\title{
Time-lag Method for Detecting Following and Leadership Behavior of Pedestrians from Mobile Sensing Data
}

\author{
Mikkel Baun Kjærgaard, Henrik Blunck, \\ Markus Wüstenberg and Kaj Grønbæk \\ Department of Computer Science, Aarhus University
}

\author{
Martin Wirz, Daniel Roggen and Gerhard Tröster \\ Wearable Computing Laboratory, ETH Zurich
}

\begin{abstract}
The vast availability of mobile phones with built-in movement and location sensors enable the collection of detailed information about human movement even indoors. As mobility is a key element of many processes and activities, an interesting class of information to extract is movement patterns that quantify how humans move, interact and group. In this paper we propose methods for detecting two common pedestrian movement patterns, namely individual following relations and group leadership. The proposed methods for identifying following patterns employ machine learning on features derived using similarity analysis on time lagged sequences of WiFi measurements containing either raw signal strength values or derived locations. To detect leadership we combine the individual following relations into directed graphs and detect leadership within groups by graph link analysis. Methods for detecting these movement patterns open up new possibilities in - amongst others - computational social science, reality mining, marketing research and location-based gaming. We provide evaluation results that show error rates down to $7 \%$, improving over state of the art methods with up to eleven percentage points for following patterns and up to twenty percentage points for leadership patterns. Our method is, contrary to state of the art, also applicable in challenging indoor environments, e.g., multi-story buildings. This implies that even quite small samples allow us to detect information such as how events and campaigns in multistory shopping malls may trigger following in small groups, or which group members typically take the lead when triggered by e.g. commercials, or how rescue or police forces act during training exercises.
\end{abstract}

Keywords-pattern recognition; crowd behavior sensing; mobile sensing; signal strength-based methods

\section{INTRODUCTION}

The vast availability of mobile phones with builtin movement and location sensors enable large-scale collection of sensor data about human movement behavior, as compared to what was previously possible with dedicated devices. However, such data only becomes valuable through methods that can process and aggregate the data to extract relevant information [7]. As mobility is a key element of many human processes and activities, an interesting class of information to extract are movement patterns that quantify how humans move, interact and group [6].

In this work we target the detection of two common pedestrian movement patterns: following relations and group leadership [2], [6]. A following pattern involves two persons and describes a relation between them, where one person is following and the other is leading. The following pattern occurs when a leading person's movement continuously prescribes the movement of a following person. A group leadership pattern is a quasi global pattern for a group of persons: It is present when a leader person's movement prescribes the movement of other persons but no other persons' movement prescribes the movement of the leader person. Both patterns are temporally and spatially constrained in so far as the persons have to be no further apart than $\delta$ meters at the same point in time.

The detection of following and leadership patterns enable new analysis methods within areas such as reality mining [7], computational social science [5], emergency research and marketing research [11], and provides new primitives for pervasive computing and location-based games. Firstly, for computational social sciences and reality mining to extract patterns among moving coworkers including staff at hospitals, caretakers in large buildings and workers in warehouses. The detected patterns provide quantitative statistics about the relationship among co-workers. Furthermore, compared to mere proximity information the following and leadership patterns can reveal asymmetries among co-worker relationships [1]. For instance, one can deduce from such patterns, whether people work independently or in teams and who leads the work. This information can then e.g. be compared with the intended working structure and help identify problems that when solved might provide e.g. gains in efficiency [23]. The clues extracted from movement and location data might also be correlated with clues extracted from audio or video data [20] or communication patterns among co-workers [7]

Secondly, there are great and growing interests within marketing research to use movement data to better understand people's shopping behavior. However, methods for properly analyzing the data are lacking [11]. An important and so far less explored aspect is the impact of social interactions [11]. One example is the detection of co-shopping behaviors [9] for which the methods proposed in this paper would enable new forms of analysis, correlating following and leadership patterns with e.g. the shopping behavior of family members, product placements and store layouts. 
Finally, the detection of following and leadership patterns also provides new primitives for pervasive computing and location-based games. We will later present an evaluation of our method in the context of a multi-person location-based evader game building on these primitives.

Previous work on the detection of following and leadership patterns in a spatio-temporal sense from movement and location data is mainly limited to the work of Andersson et al. [2], which focuses on computational efficient detection of such patterns from planar location data. They evaluate their geometric algorithm on synthetic data mimicking outdoor animal movements. However, as most humans spend a lot of their day indoors it is for many use-cases crucial to provide methods that are also applicable in multi-story buildings.

Previous work on visual scene understanding has addressed some elements of the problem mainly focusing on following patterns [17]. However, visual approaches are hampered by their need for images with a good coverage of the scene which is particular hard to satisfy in indoor areas. Furthermore, as the identity of the subjects are often unknown, vision-based methods are not applicable in several of the above scenarios. To capture non-spatio-temporal notions of leadership both audio and visual methods have been applied, e.g., by analyzing audio and visual clues during a conversation [20].

Focusing on indoor environments, there have been several works focusing on further movement patterns utilizing various sensor modalities, for example, co-moving, proximity, flocking, user behavior modeling, crowd density and group structure detection, but not following or leadership patterns. Chandrasekaran et al. [3] proposed methods for detecting co-moving devices from correlation features of WiFi signal strength measurements and later applied such methods for speed estimation [4]. Several works have utilized Bluetooth [1], [7], [8] for proximity detection. Eagle et al. [7] proposed methods for modeling users' behavior from such data and Do et al. [5] proposed methods for utilizing it for building probabilistic models of the latent group structures. Efstratiou et al. [8] used Bluetooth data to detect social interactions in a group and Adams et al. [1] used Bluetooth and GPS data to understand proximity and then model user behavior as rhythms of place visits and social interactions. Phung et al. [19] extended this work to also consider WiFi readings in an indoor setting to quantify user rhythms. Weppner et al. [22] used Bluetooth to estimate the density of a crowd. Krumm et al. [16] used WiFi measurements for detecting proximity for their NearMe system. WiFi measurements have also been utilized for flock detection [14], [15]. However, no work has so far targeted the detection of following and leadership patterns. Furthermore, technologies, e.g., Bluetooth that can only detect proximity among moving subjects are not applicable for the problem as they cannot detect who is following who - only that the two targets are in proximity.

In this paper, we propose methods identifying following

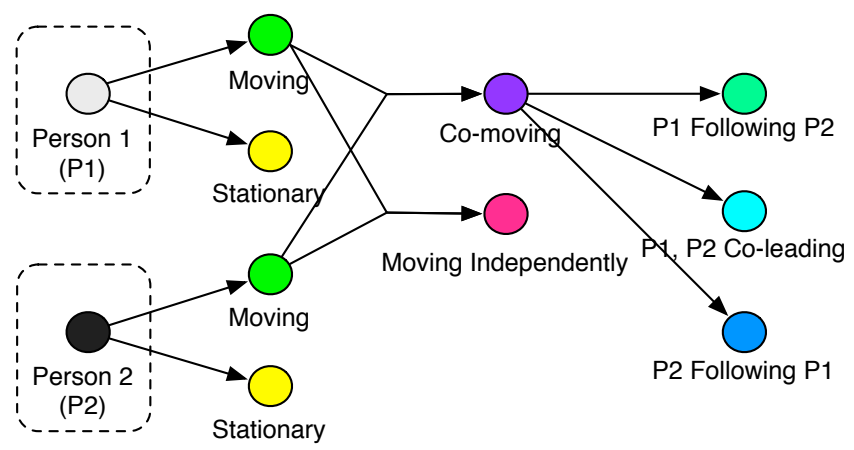

Figure 1. Activity classification states for detecting following patterns.

patterns using machine learning algorithms on features derived by using similarity metrics on time lagged sequences of mobile sensing measurements, e.g., raw WiFi signal strength and acceleration measurements. To detect leadership we first combine the detected pairwise following relations into directed graphs and then subsequently perform a graph link analysis. We provide evaluation results showing low error rates and a clear improvement over state of the art for data sets from both a scripted and an unscripted experiment, conducted in two different multi-story buildings.

The proposed methods support a fine-grained real-time analysis, that can detect following and leadership relations, and evolution thereof, in indoor environments of various spatial layouts. Our results imply, that for instance, when a crowd coming from a transit area diffuse into an open space, and smaller groups are heading in different directions, then our methods will be superior in identifying and analyzing these groups w.r.t. following and leadership patterns. Thus, they allow, e.g. to measure the effect of a commercial or an announcement in the transit area. Also, they allow for quick identification of leading characters in groups, when arriving at an open space-e.g. they allow to conclude that the teenage kids who saw a toy commercial started leading the family to the toy store. Another example is the training or evaluation of rescue teams' and police forces' actions in large multi-story public buildings, offices or industrial plants; here, our methods offer information about whether forces stuck to prescribed leadership patterns, or which events triggered new following and leadership patterns, or whether there was panic behavior, characterized by weak or highly volatile following relationships? With such accurate, fine grained, and prompt analysis, e.g. without cumbersome video analysis, the learning benefits from such training or evaluation will be much more substantial and immediate.

\section{Overview of Proposed Method}

In the following, we present the methods we propose for detecting following and leadership patterns among pedestrians, and relying on mobile sensing data ,primarily on WiFi signal strength measurements, from the pedestrians' 

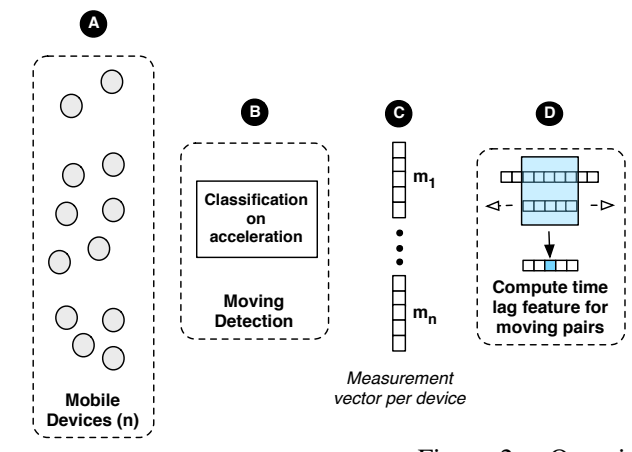
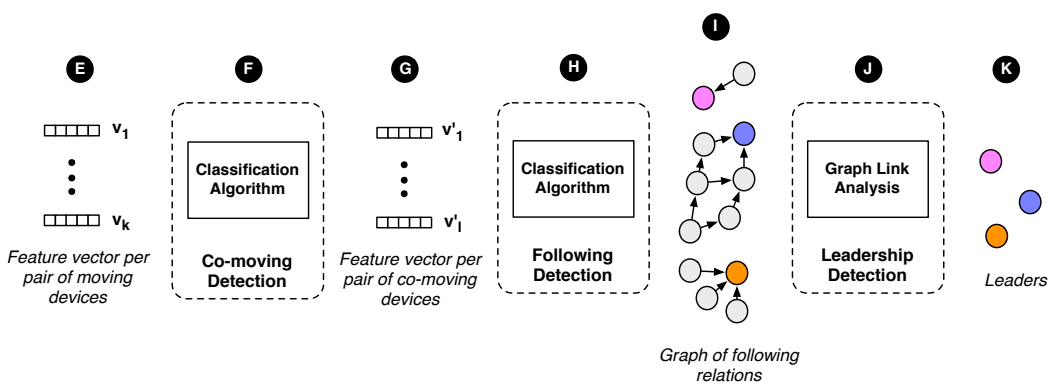

Figure 2. Overview of the method for following and leadership pattern detection.

mobile devices.

For detecting following and leadership patterns, we classify people's movement behavior according to a set of states. For two persons $\mathrm{P} 1$ and $\mathrm{P} 2$ potentially following each other, the different states in the set are shown in a classification hierarchy in Figure 1: For following detection, the first step of the hierarchy is to classify each person as moving or stationary. If the two persons in question are both moving, they are further classified with regards to if they are co-moving or moving independently. If the persons are co-moving, it is then considered which of the following three situation applies: Is P1 following P2, or P2 instead following P1, or are they co-leading each other. The co-leading state describes the situation where persons are walking side by side. We argue that the term co-leading more naturally characterizes this situation than co-following. Note also, that the the states stationary and moving independently could be further subclassified into activities such as meeting, not in proximity or passing, but this is beyond the scope of this work. For detecting the leadership pattern, we reuse the following pattern classifications by combining the pairwise following relations into directed graphs that are the basis for classifications of leadership, as will be detailed in Section IV-B.

Figure 2 shows the steps of the proposed method for detecting following and leadership patterns using mobile sensing measurements from peoples' mobile devices. We assume that a sensing service will be deployed on people's mobile devices through existing distribution channels, e.g., as part of a mobile application for a scientific study or a location-based game, or as a system service (A). When requested, the $n$ mobile devices will each measure acceleration of the device and the signal strength of nearby WiFi access points. The measurements are processed and a standard threshold-based classification on acceleration measurements is used to detect if a device is moving or stationary (B). The services on the devices $1, \ldots, n$ will send the signal strength measurements to a remote service when detected to be moving as measurement vectors $m_{1}, \ldots, m_{n}$. The remote service might be implemented as a web service running on a cloud platform (C). For each feature, the signal strength measurements are processed for pairs of devices to calculate time lagged similarity features, based on raw signal strength measurements or derived location measurements using location fingerprinting [13] (D). For each feature space the feature values are gathered in feature vectors $v_{1}, \ldots, v_{k}$ where $k$ is the number of device pairs, where both devices are moving (E).

The feature vectors are given to a classification algorithm that considers for each device pair if they are co-moving or not (F). For the co-moving devices their feature values are gathered in vectors $v_{1}^{\prime}, \ldots, v_{l}^{\prime}$ where $l$ is the number of co-moving device pairs $(\mathrm{G})$. Each vector is processed to classify it with respect to following relationships. These classifications can both be output as final results or be used for leadership detection (H). For leadership detection the pairwise following relations are combined into directed graphs (I). Leadership is detected using graph link analysis (J). The final output consists of devices classified with respect to the leadership pattern $(\mathrm{K})$. In the following we will discuss the steps in more detail.

\section{Time Lagged Similarity Features}

The proposed method for movement pattern detection requires an algorithm for binary classifying, for each pair of devices, whether they are co-moving, as sketched in the last section, see step (F) in Figure 2. In this section we introduce time-lagged similarity features and propose these as the basis for such a classification algorithm.

- enable analysis of (snapshots of) signal measurements or derived location measurements to detect movement patterns, even from non-spatial data.

- enable following detection via the intuition that if a device $b$ is following another device $a$, then observations of signal strength measurements or location coordinates for $b$ are expected to appear "delayed" with a time lag, when comparing them to the measurements from $a$.

- achieve the capturing of similarity of multi-dimensional signal measurements or derived location measurements for various time shifts via (one of two variants of) the following general scheme:

1) compute similarity, either i) for each data dimension, using classic time series metrics (such as cross correlation) or pattern recognition algorithms (Dynamic Time Warping), or ii) for each time step, using distance 


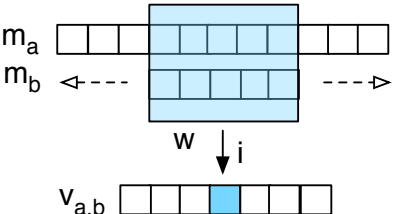

Figure 3. Feature vector $v_{a, b}$ computed for device $a$ and $b$ over a time window $w$ within the minimum and maximum lag $z$.

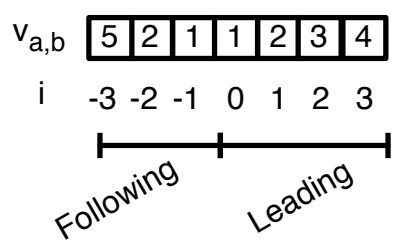

Figure 4. Feature vector $v_{a, b}$, time lag indices and mapping to following or leading classifications.

metrics (such as Euclidean distance) or similarity coefficients for sets (such as the Tanimoto Coefficient).

2) combine similarity values computed for i) individual dimensions or ii) time steps by computing average values.

We argue to use time-lagged similarity features instead of purely spatial but non-temporal features, since the latter are not able to capture temporal aspects of people's movements: For instance, Andersson et al. [2] have proposed to define the following pattern, as occurring, whenever a (leading) device is observed in a two dimensional wedge (of a given width and radius, e.g. in a half-circle) in front of another (i.e., a following) device. However, especially in restricted spaces such as indoor environments this characterization of the following pattern breaks down in many situations, such as when making frequent turns either when walking down a staircase, when walking through a crowded room, or when walking in front of one another but on different floors. In comparison, similarity features computed over a time window can operate on the similarity of movement and can thus capture following patterns also in the above situations. Furthermore, using such temporal similarity features allows to utilize also other than purely spatial data for following detection, e.g. observed signal strengths.

Formally, for a device $a$ we define $m_{a_{t}}$ as the measurements for the time step $t$. The methods presented here assume a measurement to be either a set of signal strength measurements for observable access points or a location coordinate triplet, derived from a set of signal strength measurements using location fingerprinting [13].

Time lagged features are computed for a pair of devices $a, b$ as a vector $v_{a, b}$, where each entry is associated with a certain time lag $i \in[-z, \ldots, z]$ in seconds, where $z$ defines the range of time lags, we decided to check for. Hence, the length of $v_{a, b}$ is $2 z+1$; and for each time lag $i, v_{a, b}$ then holds a feature value, which indicates the similarity of the measurements from $a$ and $b$, when shifting those of $b$ by a time lag $i .{ }^{1}$ Figure 3 illustrates the concept, and that each feature value is computed over a time window of size $w$ over $m_{a}$ and $m_{b}$, and Figure 4 depicts an example feature vector.

\footnotetext{
${ }^{1}$ Note, that we fixed the temporal granularity within $v_{a, b}$ to 1 second solely for the sake of ease of exposition.
}

\section{A. Similarity Metrics}

To unify the handling of the similarity features, when using them within our pattern detection method as sketched above, we define them all as distance metrics-so that a value of zero denotes perfect similarity and higher values denote higher dissimilarity. Some of the features have been applied by prior work for the detection of proximity, place or speed estimation. To evaluate them, with the aim to identify the similarity metric with the best performance for our pattern detection problem, we consider: i) two distance metrics: Euclidean distance and Walking distance for location data, ii) two pattern recognition algorithms: Dynamic Time Warping and Derivative Dynamic Time Warping, iii) one time series metric: cross correlation coefficient and iv) one statistical set coefficient: Tanimoto coefficient.

For a device pair $a, b$ we compute the features for each time lag entry $v_{a, b_{i}}$ for $i \in[-z \ldots z]$ with respect to the current time $t$ over a feature window of size $w$. We define two locations or signal vectors $\mathfrak{a}=m_{a_{t}}$ and $\mathfrak{b}=m_{b_{t+i}}$. The dimensionality $d$ of these vectors stems from the spatial dimensions for location data and the number of access points observable by both devices for signal data, respectively. ${ }^{2}$

Euclidean Distance (ED): is a basic distance metric which has been extensively applied for, e.g., proximity detection [16], [19]. Given a function ed that computes the Euclidean distance per time step of two vectors, we compute the similarity of the two vectors $\mathfrak{a}$ and $\mathfrak{b}$ as follows:

$$
v_{a, b_{i}}=\frac{1}{w} \sum_{0 \leq j<w} \operatorname{ed}\left(\mathfrak{a}_{j}, \mathfrak{b}_{j}\right)
$$

Walking Distance $(W D)$ : is a more refined distance metric for indoor spaces, modeling that pedestrians cannot move through walls and floor separations [15]. Given a function wd that computes the walking distance between two coordinates per time step we compute the similarity of the two vectors $\mathfrak{a}$ and $\mathfrak{b}$ using Equation 1 with wd in place of ed.

Tanimoto Coefficient (TC): is a statistical coefficient for sets which has previously been studied for proximity detection for signal strength measurements [12]. Given a function that computes the Tanimoto coefficient tc we compute the similarity of the two vectors $\mathfrak{a}$ and $\mathfrak{b}$ using Equation 1 with $1-t c$ in place of ed.

Dynamic Time Warping (DTW): is a classic dynamic programming algorithm which has been widely used for optimal alignment of two time series, but has so far not been used for movement pattern detection from signal strength or location measurements. As part of the alignment process it computes a similarity metric as a cost function equal to the minimum-distance to an optimal alignment. The

\footnotetext{
${ }^{2}$ The signal strength measurements are preprocessed, prior to the feature calculation, by excluding measurements for weak and unstable access points and by smoothing measurements - to reduce the effect of outlierswith a running average over five seconds of measurements.
} 
algorithm can potentially compute better similarity metrics than some of the other features as it can realign data, e.g, to mask inconsistent sampling of measurements, variations in walking speed of targets or outlier measurements. This charactererstic promises thus low sensitivity to i) measurement noise that often exists in complex indoor environments, and b) to smaller variations in distance and motion, as they frequently occur between two persons in a natural following relationship.

Given a function dtw that computes the cost of the minimum-distance of the optimal alignment using DTW, we compute the similarity of the two vectors $\mathfrak{a}$ and $\mathfrak{b}$ as follows:

$$
v_{a, b_{i}}=\frac{1}{n} \sum_{0 \leq k<d} d t w\left(\operatorname{dim}_{k}(\mathfrak{a}, \mathfrak{b})\right)
$$

Derivative Dynamic Time Warping (DDTW): is an extension of DTW that computes an optimal alignment of the derivatives of two time series. Existing work has considered using DDTW to estimate speed from GSM readings [4] by analyzing the warp path produced as an output of the DDTW algorithm. Here, we apply DDTW for a different task and instead of analyzing the warp path we use the cost which is the minimum-distance to an optimal alignment. Previous work that applied DDTW argued for the superiority of DDTW because it does not compare absolute values but instead compares the rate of change of the measurements. However, e.g. in indoor environments signal strength values can change rapidly and chaotically over a few meters due to obstructions by building elements, thus constituting a potential error source for DDTW. Given a function ddtw that computes this cost we compute the similarity of the two vectors $\mathfrak{a}$ and $\mathfrak{b}$ using Equation 2 with ddtw in place of dtw.

Cross Correlation $(C C)$ : Previous work has considered, both for signal strength measurements [3] and location measurements [21], to use cross correlation for detecting co-moving. Given a function $\mathrm{cc}$ that computes the cross correlation we compute the similarity of the two vectors $\mathfrak{a}$ and $\mathfrak{b}$ using Equation 2 with $1-c c$ in place of dtw.

\section{Detecting Following AND LeAdership}

\section{A. Following Detection}

As a first step of following detection we filter out non-co-moving device pairs: To detect co-moving devices, we employ a standard machine learning classifier, based on one of the presented time lagged similarity features. The classifier is trained with labeled data of co-moving devices and of devices moving individually. The data labeled individually moving includes devices far away from each other, vertically displaced and passing each other. The resulting classifier outputs for a given pair $a, b$ of devices its classification into either co-moving or moving independently. We choose a support vector machine classifier, since these
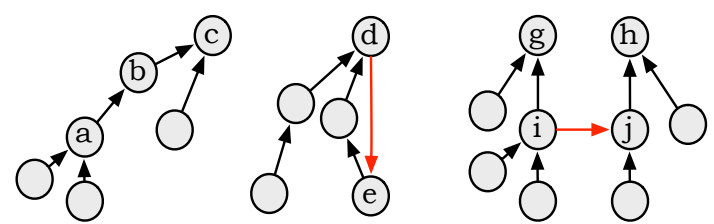

Figure 5. Example graph of detected following relations.

showed the best performance on our data sets compared to, e.g., Naive Bayes or tree-based classifiers.

For co-moving device pairs we utilize again the time lagged similarity features to detect following relations. We analyze the feature vectors $v_{a, b}$, see Figure 4 , to find the lag $i_{e s t}$, for which the highest similarity is achieved (for the measurements from the two devices), and which thus represents their most likely alignment in time. If the lag is negative, a device is classified as following, if positive as leading, and if close to zero $(+/-0.1)$ as co-leading. To extract the lag $i_{\text {est }}$ from the vector, we calculate the inverse weighted average using the similarity values as weights, where $s$ denotes the normalization factor:

$$
i_{e s t}=\frac{1}{s} \sum_{-z \leq i \leq z} \frac{1}{v_{a, b}(i)} \cdot i \text {, with } s=\sum_{-z \leq i \leq z} 1 /\left(v_{a, b}(i)\right.
$$

In the example in Figure 4, the lag is estimated to -0.23 and therefore device $b$ is classified as following a. Note, that we also evaluated to extract $i_{e s t}$ as the minimal value from $v_{a, b}$, but the weighted solution provides a 1 percentage point higher detection accuracy in our experiments.

\section{B. Leadership Detection}

As we illustrated using Figure 2, we base both the definition as well as the detection of the leadership pattern on analyzing the pairwise following relations among persons. These relations, interpreted as directed edges, constitute a graph; an example is given in Figure 5. Thus, leadership can then be detected via analyzing said graph. An apt definition of leadership is given by Andersson et al. [2], who define a leader for a given timestamp among a set of tracked persons as a person who i) has a certain minimal amount of followers, but ii) is not following any tracked person. The above definition though does not take into account some phenomena, which we aim to address in our leadership detection and which are discussed below:

Firstly, we propose to take into account the transitivity of following relations: If a person a is following a person $\mathbf{b}$ who is following a person c, we say that $\mathbf{a}$ is transitively following c. We furthermore propose to score potential leaders by counting the transitive followers for each potential leader $\mathbf{c}$ so that in the example $\mathbf{c}$ receives a higher score than a), but to do so in a weighted fashion, where the weight of a transitive follower diminishes w.r.t. to a potential follower $\mathbf{c}$ diminishes with the link distance to it. The resulting scoring scheme bears similarity to node scoring graph algorithms such as PageRank [18]. 
Secondly, our leadership detection also takes into account that the graph which it operates on may contain noise, i.e. the set of detected following relations may deviate from the actual relations - due to sensor noise or inaccurate pattern extraction. An example is given in Figure 5, where it has been erroneously detected, that $\mathbf{d}$ is following $\mathbf{e}-$ which leads to a cycle of transitive following relations in the example graph. While no leader exists in said graph component, according to Andersson's definition, we argue that a robust leadership detection should not categorically rule out identifying leaders in such cases, as depicted here with d. Note also, that such detection has no certainty about which of the edges of the cycle are erroneous, and thus cannot just eliminate cycles by removing an arbitrary edge of each cycle.

Furthermore a robust detection should also allow to relax Andersson's definition and not rule out to identify more than one leader (e.g. $g$ and $\mathbf{h}$ in Figure 5) within a (seemingly) connected component of the graph in cases, where a potentially erroneous edge connects two otherwise unconnected components of the graph (as does edge $(\mathbf{i}, \mathbf{j}$ ) in Figure 5). Note, that incorporating transitive following relations as described above may also helps addressing poor following detection accuracy, specifically in cases where some direct following relations were not detected.

Lastly, the noise-resilience of leadership detection can be increased if in the input graph each edge is assigned a weight, which resembles the confidence with which the associated following relation was detected. Such weights can then be taken into consideration by graph node scoring algorithms, such as by PageRank. In the context of leadership detection, it allows us to pay less attention or even disregard edges, which are more likely to be erroneous.

\section{Evaluation}

We evaluated for the proposed following and leadership detection methods their detection accuracy, considering the accuracy of the individual features and comparing with state of the art algorithms.

\section{A. Experiments and Data Sets}

We utilize two data sets in our evaluation. The first was collected during a scripted experiment using data from fourteen participants and the second during an unscripted experiment using a location-based game as evaluation setting with ten participants. Table I lists the properties of the individual scenes of the two experiments. As shown in Figure 6, the scripted experiment was conducted in an office building and the unscripted experiment in a lecturing building with large open spaces. Both buildings had a deployment of $\mathrm{WiFi}$ access points and we observed on average ten and eleven access points per WiFi measurement for the scripted and the unscripted experiment, respectively.

In both experiments we utilized Android phones of various models: Google Nexus, HTC Desire and Samsung
Table I

SCENES OF THE EXPERIMENT

\begin{tabular}{ll} 
Name & Description \\
\hline Scripted - Horiz. A & Subjects moving on a single floor (4:08 min). \\
Scripted - Vertical A & Subjects moving on both floors (4:27 min). \\
Scripted - Vertical B & Subjects moving on both floors (10:25 min). \\
Scripted - Vertical C & Subjects moving on both floors (7:23 min). \\
Unscripted - Horiz. A & Subjects moving on a single floor (8:40 min). \\
Unscripted - Horiz. B & Subjects moving on a single floor (6:56 min). \\
\hline
\end{tabular}
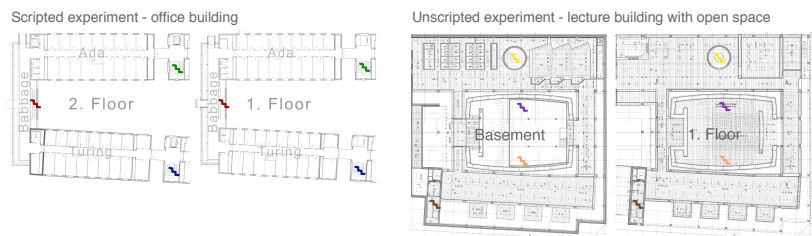

Figure 6. Building layout for two buildings with icons for staircases.

Galaxy S+. The phones ran dedicated sensor logging software to record WiFi signal strength and acceleration at the platform's default sampling rate. For the WiFi positioning we collected fingerprints throughout the buildings in the scripted experiment in a 1.5 meter grid on two floors and in the unscripted experiment in a 3.0 meter grid on a single floor.

The scripted experiment was conducted according to a predefined script and monitored with video cameras and manual annotation in order to obtain a ground truth against which the method's performance can be evaluated. During each scene, following relations and the subjects showing leadership behavior are changing several times. For obtaining ground truth we analyzed the video to label the participants' behavior accordingly. In order to obtain recordings of natural behavior, we did not instruct the subjects on how to move or behave and only instructed them to keep their mobile phone in alternatively their left or right front trouser pocket and stay close together in their designated group (where "close" was not further specified but was observed to be around $1.5 \mathrm{~m}$ ).

The unscripted experiment was conducted using a location-based game as an evaluation setting. The game was designed as a "follower-evader" game with each subject taking the role as either a follower or an evader. Followers would score points by following an evader in close distance ("close" being specified to participants as up to 7 meters) and evaders would score points when nobody followed them. Using the method presented in this paper we would be able to implement this game without manual observation or intervention on the participant side. However, to obtain ground truth labels while collecting the data set, we designed a smartphone game application in which the participants labeled the data as part of the game. Figure 7 shows both a picture from the experiment with evaders in orange jerseys and the game application interface. The scoring scheme was designed so that the followers had to occasionally change evaders-enticed by bonus points and 


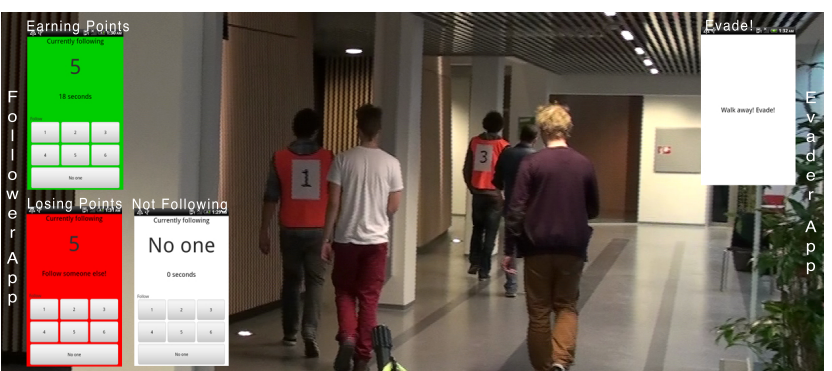

Figure 7. Game experiment including game application interface for followers and evaders.

negative points for staying with the same evader for longer than 45 seconds. This information was communicated to the participants by the game application via vibration and a color scheme. These elements were also designed to keep the players attending to the app and update the information continuously to score points. However, to minimize timing flaws in the participants' labelling, we chose to remove labels for the first seconds before/after a change and to not compute patterns for these points in time.

\section{B. Results}

Following Detection: To evaluate the proposed detection method, we compare detection results to the collected ground truth information. We compute the detection error rate of the detection individually for the two datasets, and also individually on both location and signal data for the considered feature types: Euclidean Distance (ED), Dynamic Time Warping (DTW), Derivative Dynamic Time Warping (DDTW) and Cross Correlation (CC). We evaluate the methods using several window sizes $w$ but the main results are given for $w=10$ seconds. To reflect that we for the experiments defined the spatial constraint of the patterns $\delta$ as seven meters, we set the absolute time lag maximum $z$ to seven seconds, which corresponds to the maximal temporal distance between persons in a valid following-leading relationship, when assuming a walking speed of around $1 \mathrm{~m} / \mathrm{s}$. To demonstrate that our method generalizes in the sense that it works in various environments with different building elements and access point densities, we always trained the co-moving classifiers using data from an experiment different from the one we test the method in: E.g., for the given test results for the scripted experiment, we trained with data solely from the unscripted experiment. We compare our method to two state-of-the-art detection methods. First, the method proposed by [3] for co-moving detection based on cross correlation, and secondly, the geometric method by Andersson et al. [2] for both co-moving, following and leadership pattern detection.

As co-moving detection is a necessary pre-step for the following detection, we also present results for this step in Figure 8 and 9 for the scripted and the unscripted experiment, respectively. As can be seen for both the scripted and unscripted experiment, overall the DTW

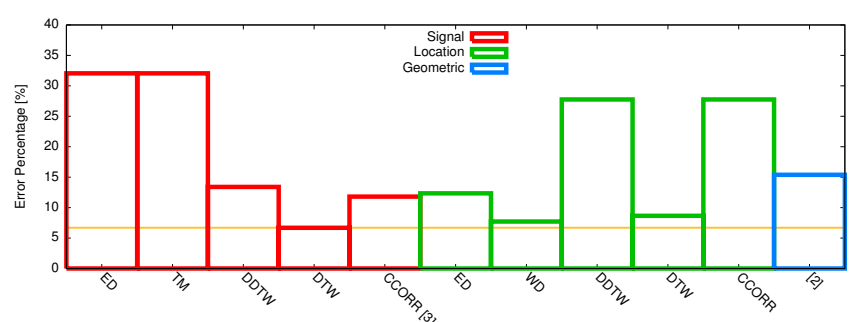

Figure 8. Error rates for co-moving detection for the scripted experiment.

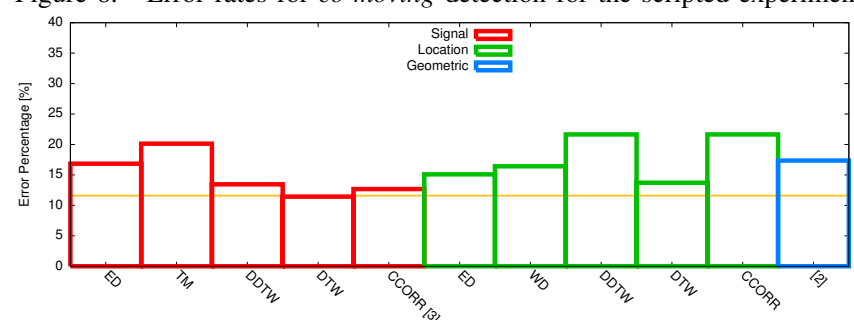

Figure 9. Error rates for co-moving detection for the unscripted experiment.

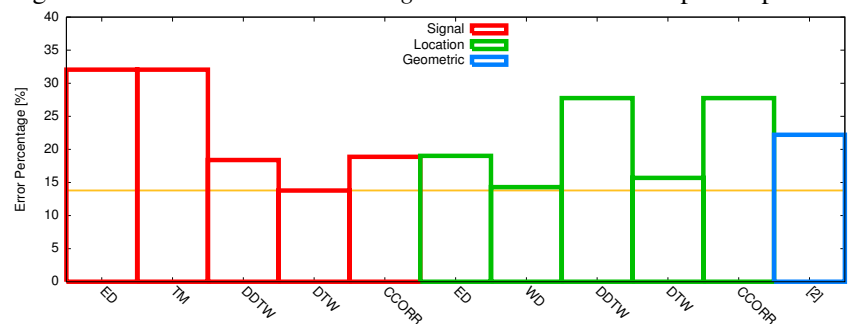

Figure 10. Following detection accuracy for the scripted experiment.

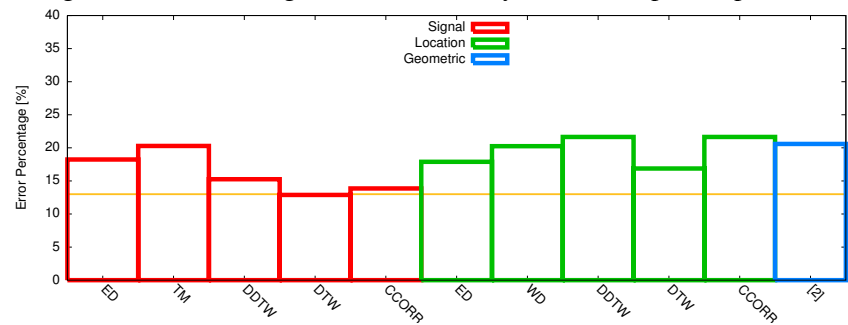

Figure 11. Following detection accuracy for the unscripted experiment. feature outperforms the other features with error rates down to $7 \%$. Applied to our data sets, it provides an error reduction of up to five percentage points when compared to [3] and up to eleven percentage points when compared to [2]. We attribute the superiority in performance, consistent over both experiments, to the DTW method's mentioned low sensitivity to data fluctuations, which occur even when the two entities are in a solid and natural co-moving relation, namely typical measurement noise as well as small fluctuations in relative motion and distance between the entities. Only for the scripted experiment exists a contender with similar performance, namely the WD feature.

Leadership Detection: The results for following pattern detection are given in Figure 10 and 11 for the scripted and unscripted experiment, respectively. Once more, the DTWbased feature provides the best performance, i.e. an error reduction of up to 11 percentage points when compared to [2], i.e. a relative error rate reduction of $45 \%$ and $37 \%$ for the scripted and unscripted experiments, respectively.

For window sizes $w$ for the feature calculation we com- 


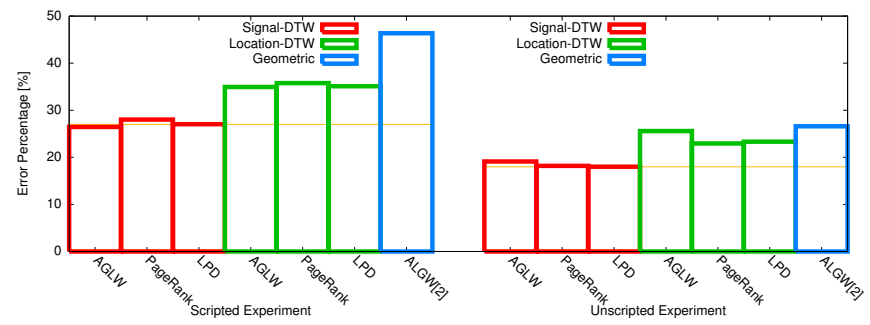

Figure 12. Leadership detection accuracy for all experiments.

puted results also for $w=5$ and $w=15 \mathrm{~s}$. We These results revealed that for the unscripted experiment a value of ten seconds gave the best results whereas for the scripted experiments a value of fifteen seconds provided an improvement of a few percentage points. This difference among the experiments we expect can be attributed to a difference in average time between change in following relations. Therefore, for the scripted experiment with less changes in the following relations a larger window size is beneficial, whereas for the unscripted experiment a smaller one is suitable. Therefore the window size is an application-specific choice and resembles a trade-off between accuracy versus detection latency.

Figure 12 compares the error rate as exhibited in the two experiments, respectively, for different leadership detection methods, namely for Andersson et al.'s method (labeled 'ALGW') [2], an instance of the PageRank algorithm [18], and our method (labeled 'LDP') using the techniques described in Section IV-B. ${ }^{3}$ In blue is shown as a baseline Andersson et al.'s method for leadership detection, showing error rates of about $46 \%$ for the scripted and $24 \%$ for the gaming experiment, respectively. Provided as input to the detection algorithm were the following relations, as detected by Andersson et al.'s method. If instead the output from our proposed following detection methods, specifically from the DTW-based method, is used as input, accuracy is improved: for the scripted experiment by 11 percentage points when running the DTW-based detection on location data, and yet by 20 percentage points when running on raw signal data instead. For the gaming experiment, the improvements are 1 and 7 percentage points, respectively. Furthermore, when now using PageRank instead of Andersson et al.'s method for graph link analysis, we see a slight improvement only in the unscripted experiment, but an increased error rate in the scripted experiment. Finally, our proposed leadership detection method generally improves over the two other graph analysis methods, but never by more than 2 percentage points. Thus, a general conclusion from the depicted evaluation results for leadership detection is that the accuracy of the latter is highly impacted by the accuracy of the utilized following pattern detection method-whereas the difference between graph link analysis methods, when applied to the same set of following relations, is comparatively small.

\footnotetext{
${ }^{3}$ The results shown for the page rank algorithm are obtained using the implementation within Networkx [10] and parameter choices, resulting in superior detection accuracy, i.e. a maximum of 200 iterations and alpha $=0.85$.
}

\section{DISCUSSION}

In the last section, we have presented and in part analyzed the results of our evaluation, which demonstrated the good performance of the proposed method. In this section, we will discuss the differences which show in the results across the data sets and for using raw signal strength data compared to using derived locations.

Results from Scripted Versus Unscripted Experiments: In regards to lowest error rates for co-moving detection, we achieve the best results in the scripted experiment, whereas the lowest error rates in the unscripted experiment are five percentage points higher. Three of the main reasons for this difference are: Firstly, the unscripted experiments were conducted in a more open spaced building rendering signal strength measurements less descriptive over similar distances. This led to more co-moving detection errors in the unscripted experiment. Secondly, the density of participants was slightly higher in the unscripted experiment. The effect of this can be observed by comparing the two unscripted scenes to a third unscripted scene where participants where allowed to utilize not one, but two floors. ${ }^{4}$ We achieved $c o$ moving error rates of $7 \%$ for this scene - considerably lower than for the scenes restricted to one floor, since the wider separation between participants enabled better co-moving detection. Thirdly, at some points in the game experiment several evaders were walking so close to each other, that some of their followers factually co-moved with not one (as labeled in the ground truth) but with multiple evaders.

Naturally, for both most of the following, as well as for the leadership detection methods, their performance is impacted by the accuracy with which the more basic patterns are detected, i.e. co-moving for following detection, and following for leadership detection. For the following detection method, though, which performs best in both experiments, we obtain slightly better accuracy improvements-both for following and for leadership detection compared to the co-moving performance -in the unscripted experiment. The main reason for this being that the participants in the scripted experiment were on average walking closer to each other than in the unscripted experiment, thereby making it potentially harder for the algorithms to identify who is following who among a pair or group of persons, who are detected as co-moving.

Using Signal vs. Location Data: For the more accurate among the evaluated following detection methods, specifically for the superior DTW-based one, error levels for signal data input are slightly lower than for location data input. ${ }^{5}$ For following detection these gains are visibly higher for the unscripted experiment, which was conducted in a more open

\footnotetext{
${ }^{4} \mathrm{We}$ did not include the data for this scene in the main results due to lack of location fingerprints for the whole area.

${ }^{5}$ This difference in accuracy is even more visible for CCORR and DDTW, but their classification performance generally profits from higher dimensionality of input data (here: number of observable access points vs. at most three spatial dimensions).
} 
spaced building part and for which the collection of location fingerprints was carried out in a less dense grid. For the detection of leadership patterns in both experiments, see Figure 12 , the accuracy gains when using signal instead of location data are even larger than for following detection. We conclude from these results that i) for following detection it becomes necessary to provide a well calibrated location system in order to match the accuracy that can be achieved from raw signal data (without any area-specific calibration), and ii) for leadership detection the latter data type may still provide superior accuracy. However, note that positioning capabilities prove useful as they allow to associate the detected movement patterns with the spatial location where they occurred -which may be relevant in various use-case scenarios.

\section{CONClusions And Future Work}

We presented a time-lag analysis-based method for accurately detecting following and leadership movement patterns. The method can operate on various kinds of mobile sensing data, e.g. on bare bones radio signal strength or on derived location data. We showed the superior accuracy of the method by comparing against several approaches in two experimental settings in different indoor environments. Based on our evaluation, we also discussed factors impacting detection accuracy to be expected in different scenarios. The promising results suggest that the proposed method should be further tested in the outlined application domains in order to enable new applications based on real-time analysis of following and leadership behavior of pedestrians.

However, there remain challenges for future research, e.g. in interpreting the semantics of detected movement patterns in relation to the build environment and social and cultural differences. Such differences are hard to capture for the proposed time-lag method alone; therefore, one could enrich the method with the detection of spatial characteristics or clues from other modalities such as audio and video when available, in order to achieve a more context-tailored and more differentiated, and thereby more accurate, pattern detection.

\section{ACKNOWLEDGEMENTS}

This work is supported by the Danish Council for Strategic Research for the project EcoSense, the FP7 ICT Future Enabling Technologies Programme under grant agreement No 231288 (SOCIONICAL) and by a grant from the Carlsberg Foundation.

\section{REFERENCES}

[1] B. Adams, D. Q. Phung, and S. Venkatesh. Sensing and using social context. TOMCCAP, 5(2), 2008.

[2] M. Andersson, J. Gudmundsson, P. Laube, and T. Wolle. Reporting leaders and followers among trajectories of moving point objects. GeoInformatica, 12(4):497-528, 2008.

[3] G. Chandrasekaran, M. A. Ergin, M. Gruteser, R. P. Martin, J. Yang, and Y. Chen. Decode: Exploiting shadow fading to detect comoving wireless devices. IEEE Trans. Mob. Comput., 8(12):1663-1675, 2009.
[4] G. Chandrasekaran, T. Vu, A. Varshavsky, M. Gruteser, R. P. Martin, J. Yang, and Y. Chen. Tracking vehicular speed variations by warping mobile phone signal strengths. In In PerCom, pages 213-221, 2011.

[5] T. M. T. Do and D. Gatica-Perez. Contextual grouping: Discovering real-life interaction types from longitudinal bluetooth data. In In MDM, pages 256-265, 2011.

[6] S. Dodge, R. Weibel, and A.-K. Lautenschütz. Towards a taxonomy of movement patterns. Information Visualization, 7(3-4):240-252, 2008.

[7] N. Eagle and A. Pentland. Reality mining: sensing complex social systems. Personal and Ubiquitous Computing, 10(4):255-268, 2006.

[8] C. Efstratiou, I. Leontiadis, M. Picone, K. K. Rachuri, C. Mascolo, and J. Crowcroft. Sense and sensibility in a pervasive world. In In Pervasive, pages 406-424, 2012.

[9] S. Grossbart, L. Carlson, and A. Walsh. Consumer socialization and frequency of shopping with children. Journal of the Academy of Marketing Science, 19:155-163, 1991.

[10] A. A. Hagberg, D. A. Schult, and P. J. Swart. Exploring network structure, dynamics, and function using NetworkX. In In SciPy, pages 11-15, 2008.

[11] S. K. Hui, P. S. Fader, and E. T. Bradlow. Path data in marketing: An integrative framework and prospectus for model building. Marketing Science, 28(2):320-335, Mar. 2009.

[12] D. H. Kim, Y. Kim, D. Estrin, and M. B. Srivastava. Sensloc: sensing everyday places and paths using less energy. In In SenSys, pages 43-56, 2010.

[13] M. B. Kjærgaard and C. V. Munk. Hyperbolic location fingerprinting: A calibration-free solution for handling differences in signal strength. In In PerCom, pages 110-116, 2008.

[14] M. B. Kjærgaard, M. Wirz, D. Roggen, and G. Tröster. Detecting pedestrian flocks by fusion of multi-modal sensors in mobile phones. In In UbiComp '12, pages 240-249. ACM, 2012.

[15] M. B. Kjærgaard, M. Wirz, D. Roggen, and G. Tröster. Mobile Sensing of Pedestrian Flocks in Indoor Environments using WiFi Signals. In In PerCom, 2012.

[16] J. Krumm and K. Hinckley. The nearme wireless proximity server. In In UbiComp, pages 283-300, 2004.

[17] N. Oliver, B. Rosario, and A. Pentland. A bayesian computer vision system for modeling human interactions. IEEE Trans. Pattern Anal. Mach. Intell., 22(8):831-843, 2000.

[18] L. Page, S. Brin, R. Motwani, and T. Winograd. The pagerank citation ranking: Bringing order to the web. Technical report, 1999.

[19] D. Q. Phung, B. Adams, K. Tran, S. Venkatesh, and M. Kumar. High accuracy context recovery using clustering mechanisms. In In PerCom, pages 1-9, 2009.

[20] D. Sanchez-Cortes, O. Aran, M. S. Mast, and D. GaticaPerez. A nonverbal behavior approach to identify emergent leaders in small groups. IEEE Transactions on Multimedia, 14(3-2):816-832, 2012.

[21] T. Shirabe. Correlation analysis of discrete motions. In In GIS, pages 370-382. Springer, 2006.

[22] J. Weppner and P. Lukowicz. Bluetooth based collaborative crowd density estimation with mobile phones. In In Percom). IEEE, 2013.

[23] L. Wu, B. N. Waber, S. Aral, E. Brynjolfsson, and A. Pentland. Mining face-to-face interaction networks using sociometric badges: Predicting productivity in an it configuration task. In In ICIS, 2008. 